\title{
Biomarker testing in non-small cell lung cancer: to move forward with quality
}

\author{
Rolf Stahel
}

$\mathrm{W}^{\mathrm{i}}$ ith the recognition of the molecular heterogeneity of non-small-cell lung cancer and -importantly- the emerging possibilities of personalised therapy for patients with advanced disease, biomarker testing in addition to conventional pathology has become a central issue. If decisions on personalised therapy now rely on the determination of biomarkers, then we have to be diligent in providing high-quality testing for the community. In order to really make an impact for patients, it has become evident that closer collaboration and better mutual understanding of the respective issues between pathologists and oncologists are required. It is in this spirit that the Guidelines for Biomarker Testing in advanced non-small cell lung cancer have been elaborated jointly by the Spanish Society for Medical Oncology and the Spanish Society for Pathology [1].

The ESMO consensus statement elaborated in 2010 and published the following year recommends EGFR somatic mutation testing to identify patients with nonsmall-cell lung cancer eligible for first-line treatment with an EGFR TKI. Testing should be done in never smokers or former light smokers or patients whose tumour has a nonsquamous cell histology [2]. A 2012 update of the ESMO consensus statement will also address the issue of ALK testing.

The testing of a biomarker alone is important, but so is the definition of the entire process, from tissue sampling until the final reporting of the results, as the clinician uses this information to make his decision. This was described in a European Workshop for EGFR testing in non-smallcell lung cancer which took place 2 years ago [3]. In addition to defining a standardised process, timing was felt to be of great importance and it was the wish of the participants that test results be available within one week.

\section{R. Stahel ( $\square)$}

Clinic of Oncology

University Hospital

CH-8091 Zürich, Switzerland

e-mail: rolf.stahel@usz.ch
The key challenge for biomarker testing is the availability of sufficient tissue, as often only small biopsy samples are available from patients with metastatic disease. This has been addressed by a working group of European pathologists who developed algorithms for the diagnostic process including immunohistochemistry and molecular analysis in small tissue samples [4].

The guidelines for biomarker testing published by the Spanish Societies also address the methodologies of EGFR testing. It is important to distinguish two principals of EGFR testing. The first is screening technologies to detect all EGFR mutations, including new mutations. The second is targeted technologies to detect specific known mutations. The former are currently more widely available, but are less sensitive and need more expertise and are more labour intensive. The latter are more sensitive and generally less time consuming, but do not detect all mutations, generally need to be processed in batches and are more expensive. However, with the rapidly evolving technologies there will be increasing use of multiplex genotyping giving information about several genes of interest [5]. Also, in the not too distant future we can expect methods for genomic analysis to enter clinical investigation and, later, clinical practice [6].

How to best investigate for ALK translocations is a matter of ongoing research. Currently the break-apart FISH technology is seen as standard. In the future this might be complemented by screening with immunohistochemistry and multiplex PCR methods.

The National Spanish Consensus statement on biomarker testing in advanced non-small-cell lung cancer is a great example of how to move forward. The key to success will be emphasis on continuous external quality control to ensure that the written reports going out the treating physicians are reliable, as only then patients can profit from personalised therapy [2]. Guidelines the for external quality assurance programmes for molecular pathology are currently being elaborated in a collaboration between European Societies and the Italian Medical Oncology Society (AIOM). 


\section{References}

1. Garrido P, de Castro J, Concha Á et al (2012) Guidelines for biomarker testing in advanced non-small-cell lung cancer (NSCLC). A national consensus of the Spanish Society of Medical Oncology (SEOM) and the Spanish Society of Pathology (SEAP). Clin Transl Oncol 14:338-349

2. Felip E, Gridelli C, Baas P et al; panel members (2010) Metastatic non-small cell lung cancer: consensus on pathology and molecular tests, first line, second line, and third line. 1st ESMO Consensus Conference in Lung Cancer, Lugano 2010. Ann Oncol 22:1507-1519

3. Pirker R, Herth F, Kerr K et al; European EGFR Workshop (2010) Consensus for EGFR mutation testing in non-small cell lung cancer. Results from a European Workshop. J Thorac Oncol 5: 1706-1712

4. Thunnisson E, Kerr, K, Herth F et al (2012) The challenge of NSCLC diagnosis and predictive analysis on small samples. Practical approach of a working group. Lung Cancer 76:1-18

5. Sequist LV, Heist RS, Shaw AT et al (2011) Implementing multiplexed genotyping on non-small cell lung cancer in routine clinical practice. Ann Oncol 22:2616-2624

6. Polity K, Lynch TJ (2012) Two sides of the same coin: EGFR exon 19 deletions and insertions in lung cancer. Clin Cancer Res [Epub ahead of print] 